\title{
Molecular Docking of Polycyclic Aromatic Hydrocarbons as Potentially Carcinogenic Molecules Through Binding with Aryl Hydrocarbon Receptor
}

\author{
Mas Adam Lukman Chaubah ${ }^{1 *}$, Brenda Wilhelmina Bontes ${ }^{1,2}$, Naqiyah Afifah Mulachelah ${ }^{1}$ \\ ${ }^{1}$ Department of Biology, Faculty of Mathematics and Natural Sciences, Brawijaya University \\ Jalan Veteran Malang East Java Indonesia 65145 \\ ${ }^{2}$ HAN University of Applied Science, Institute of Life Sciences, Nijmegen, The Netherlands
}

Submission: August 2019; Revised: September 2019: Accepted: October 2019

*Corresponding author: Mas Adam Lukman Chaubah; e-mail: adamchaubah@gmail.com

\begin{abstract}
Aryl hydrocarbon Receptor (AhR) can activate gene target regulation through transcription, activation, or deactivation. Ligands for the AhR are mostly aromatic hydrocarbons. One of them is Polycyclic Aromatic Hidrocarbons $(P A H s)$. These compounds are naturally found everywhere and are strong carcinogens. The purpose of this study was to perform a molecular docking analysis of three PAHs compounds (BaA, BaP, and PA) towards AhR to observe the strongest interaction in which could potentially lead to carcinogenesis. In this research, we retrieved the strongest three of the PAHs types: Benz[a]Anthracene (BaA), Benzo[a]Pyrene (BaP), and Phenanthrene (PA) from PubChem database. PyRx was used to minimize the ligands energy. Protein model of AhR (ID: 5NJ8) was obtained from PDB database. Discovery Studio Client 3.5 was used to remove water molecules and ligands attached to AhR. We interacted each ligand to the receptor by HEX 8.0.0 and visualized using Discovery Studio Client 3.5. We found that BaP, followed by BaA and $P A$, had the strongest interaction towards AhR. It indicated that BaP had a higher risk leading to cancer with more adverse effects compared to the BaA and PA interaction to AhR.
\end{abstract}

Keywords: Aryl hydrocarbon Receptor; Polycyclic Aromatic Hydrocarbons; carcinogen; molecular docking

\section{INTRODUCTION}

Aryl hydrocarbon Receptor (AhR) is a nucleat/steroid receptor of xenobiotics which can activate gene target regulation through transcription, activation, or deactivation [1]. When AhR is activated, it could facilitate the biotransformation and elimination of compounds which goes into the body from the air, water, and food. Xenobiotics, the ligands for the AhR, are mostly aromatic hydrocarbons. These aromatic hydrocarbons groups are for instance: Halogenated Aromatic Hydrocarbons (HaHs), Polychlorobiphenyls (PCBs), and Polycyclic Aromatic Hydrocarbons (PAHs) [2].

PAHs consist of carbon and hydrogen atoms in two or more aromatic rings. These compounds are naturally everywhere, mainly from volcanoes, forest fires, burning coal, car exhaust, and cigarette smoke [1]. Exposure to this compound is known to cause irritation of the eyes and breathing passages, as well as cancer due to its carcinogenic properties [3]. WHO reported that cancer is the second leading cause of death in the world. PAHs are strong carcinogenic compounds, which consist of many types. Some of these types have a greater risk of causing cancer than others. Based on this reason, we can comprehend the prominent of this research to be done.

In this research, we investigated the strongest three of the PAHs types: Benz[a] Anthracene (BaA),
Benzo[a]Pyrene (BaP), and Phenanthrene (PA). BaA consists of four benzene rings. $\mathrm{BaP}$ consists of five benzene rings. Meanwhile, PA consists of three benzene rings [1]. We are compared which of them that can act as cancer factor by performing in silico docking analysis of AhR (as a receptor) with the selected PAHs (as ligands). This was done by defining their bonding affinity, binding location prediction, or interactions.

\section{METHODS}

\section{Ligands and Protein Receptor Preparation}

The ligands such as BaA (CID: 5954), BaP (CID: 2336), and PA (CID: 995) were obtained from PubChem database as 3D-SDF format. PyRx was used to minimize the ligands energy and convert to PDB format. The protein model of AhR (ID: 5NJ8) was downloaded from PDB database. Discovery Studio Client 3.5 was used for preparing the receptor. We removed the water molecules and ligands attached to AhR.

\section{Docking of Ligand-Protein and Visualization}

HEX 8.0.0 was used to dock each ligand to the receptor. We predicted the possible interaction and energy binding of $\mathrm{AhR}$ with $\mathrm{BaA}, \mathrm{BaP}$, or PA (Table 1). The result was then visualized using Discovery Studio Client 3.5. 
Chaubah, M.A.L., Bontes, B.W., Mulachelah, N.A. - Molecular Docking of Polycyclic Aromatic Hydrocarbons as Potentially

Table 1. Docking combinations between the receptor and ligand which was performed using HEX 8.0.0.

\begin{tabular}{|c|c|c|c|c|c|}
\hline Ligand & $\begin{array}{c}\text { Energy } \\
\text { (kcal/mol) }\end{array}$ & Name & $\begin{array}{c}\text { Distance } \\
\text { (囚) }\end{array}$ & Category & Types \\
\hline \multirow{6}{*}{$\mathrm{BaA}$} & \multirow{6}{*}{-232.9} & :LIG1 - D:ARG143 & 4.84732 & Hydrophobic & Pi-Alkyl \\
\hline & & :LIG1 - D:ARG143 & 3.88484 & Hydrophobic & Pi-Alkyl \\
\hline & & :LIG1 - B:PRO327 & 4.63696 & Hydrophobic & Pi-Alkyl \\
\hline & & :LIG1 - D:ARG143 & 4.31288 & Hydrophobic & Pi-Alkyl \\
\hline & & D:ARG143:HE - :LIG1:H & 1.23988 & Unfavourable & Unfavourable Bump \\
\hline & & D:GLY144:HC - :LIG1:C & 1.92828 & Unfavourable & Unfavourable Bump \\
\hline \multirow{10}{*}{$\mathrm{BaP}$} & \multirow{10}{*}{-230.6} & A:ASP48:OD2 - :LIG1 & 3.47085 & Electrostatic & Pi-Anion \\
\hline & & A:ASP48:OD2 - :LIG1 & 3.37140 & Electrostatic & Pi-Anion \\
\hline & & A:ASP48:OD2 - :LIG1 & 3.40971 & Electrostatic & Pi-Anion \\
\hline & & :LIG1 - A:ALA51 & 4.71448 & Hydrophobic & Pi-Alkyl \\
\hline & & :LIG1 - A:ALA51 & 4.32373 & Hydrophobic & Pi-Alkyl \\
\hline & & :LIG1 - C:LEU227 & 4.79783 & Hydrophobic & Pi-Alkyl \\
\hline & & :LIG1 - C:LEU227 & 5.41073 & Hydrophobic & Pi-Alkyl \\
\hline & & :LIG1 - A:ALA51 & 5.13129 & Hydrophobic & Pi-Alkyl \\
\hline & & :LIG1 - A:LYS66 & 4.96237 & Hydrophobic & Pi-Alkyl \\
\hline & & :LIG1 - A:VAL69 & 5.43472 & Hydrophobic & Pi-Alkyl \\
\hline \multirow{4}{*}{ PA } & \multirow{4}{*}{-191.93} & A:LYS66:NZ - :LIG1 & 3.98893 & Electrostatic & Pi-Cation \\
\hline & & A:LYS66:NZ - :LIG1 & 4.10921 & Electrostatic & Pi-Cation \\
\hline & & :LIG1 - A:LYS66 & 4.46286 & Hydrophobic & Pi-Alkyl \\
\hline & & :LIG1 - A:LYS66 & 3.78527 & Hydrophobic & Pi-Alkyl \\
\hline
\end{tabular}

\section{RESULTS AND DISCUSSIONS}

The result of docking between Benz[a]Anthracene (BaA) towards AhR showed 6 interactions in total. The types of interactions were 4 non-bond interactions and 2 unfavourable nonbonds. The non-bond interactions indicated the Pialkyl interaction type which was categorized as a hydrophobic interaction. Three of the hydrophobic interactions were found between $\mathrm{BaA}$ and arginine143 on the PAC domain of AhR, meanwhile one hydrophobic interaction was found between $\mathrm{BaA}$ and proline-327 on the PAS 1 (Per-Arnt-Sim 1) domain of AhR (Fig. 1). Each distance from the smallest to the largest was $3.88 \AA, 4.31 \AA$, $4.63 \AA$, and $4.84 \AA$. Two unfavourable non-bond types yield distances within $1.24 \AA$ to $1.93 \AA$, which occured in the steric region. Van der Waals force usually contributes significantly to the cohesion energies and interfacial energies of solids and liquids. In these cases, Van der
Waals force determines the interactions occuring at interatomic spacings. This is where distance of the bonds and the energy are crucial [4].

The docking of Benzo[a]Pyrene (BaP) and AhR showed that there were 10 non-bond interactions. The non-bond interaction indicated that there were 3 bonds in the form of Pi-anions, all of the them were categorized as electrostatic interactions between the $\mathrm{BaP}$ and aspartic acid-48 on bHLH (basic helix-loophelix) domain of AhR (Fig. 1). The other 7 non-bond interactions were Pi-Orbitals type.They were categorized as hydrophobic interactions. Five of the hydrophobic interactions were found between $\mathrm{BaP}$ and alanine-51, between $\mathrm{BaP}$ and lysine-66, and once between $\mathrm{BaP}$ and valine- 69 on the bHLH domain of AhR (Fig. 1). Meanwhile two hydrophobic interactions were found between $\mathrm{BaP}$ and leusine227 on the PAS 2(Per-Arnt-Sim 2) domain of AhR (Fig. 1). The longest distance of the non-bond interactions was $5.435 \AA$ (hydrophobic), while the closest distance was $3.371 \AA$ (electrostatic). 


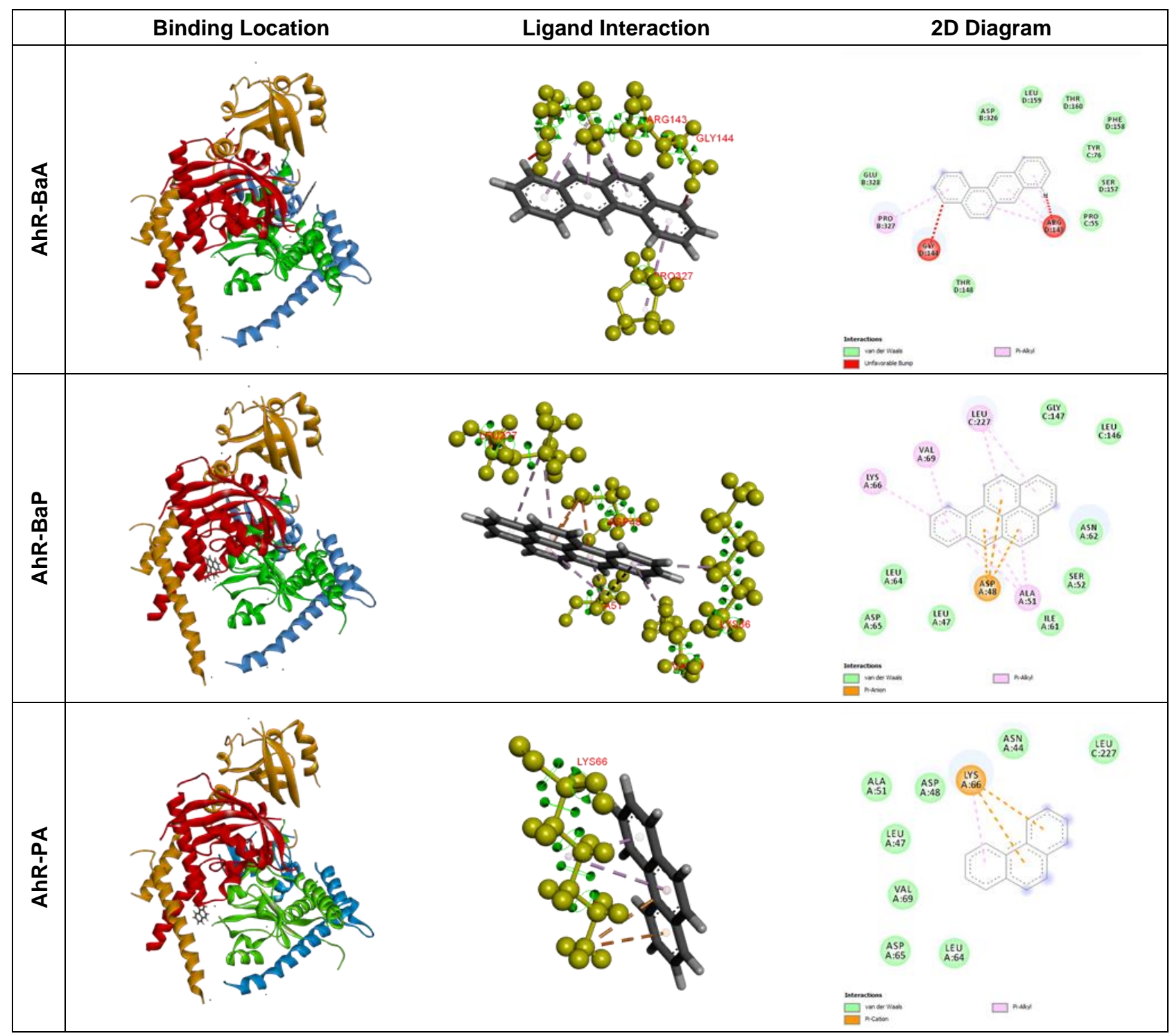

Figure 1. The 3D and 2D structures of docking between BaA (top), BaP (middle), and PA (bottom) towards Aryl hydrocarbon Receptor (AhR). Binding location of the ligands is shown on the left column; AhR domains are shown in different colour as following: bHLH (red), PAS 1 (orange), PAS 2 (green), and PAC (blue). Middle column shows the ligand binding interactions between the ligand and amino acids of AhR

Phenanthrene (PA) and AhR complex established 4 non-bond interactions on the bHLH domain of AhR (Fig. 1). The non-bond interactions showed that there were 2 bonds, between lysine-66 of AhR and PA, which was categorized as electrostatic interactions. The other 2 bonds found were between PA and lysine- 6 of AhR. Both of them were classified as hydrophobic Pi-alkyl interactions.

Based on our docking results, most of the interactions were hydrophobic interactions. Interactions from the strongest occurred, consecutively, in $\mathrm{BaP}, \mathrm{BaA}$, and $\mathrm{PA}$. The noncovalent interactions (hydrogen and halogen bonding, van Der Waals forces, etc.) use much lower energy than the covalent one. However, regarding their multiplicity and facile transformation, the overall influence of a reaction can be decisive. The hydrogen bonds influence the reactivity of the substrate in various ways. For example, an oxygen atom of a carbonyl group of a thioketone requires a strong bifurcated hydrogen bonding, the electrophilic character of the corresponding carbon increases toward the nucleophile attack, compared with a simple hydrogen bonding [5]. The same case can occur in this study, the hydrophobic interactions, combined with the electrostatic can produce a strong impact.

$\mathrm{BaP}$ and PA binds mostly onto the basic helixloop-helix (bHLH) which functions as the DNA binding region of AhR [6]. The strong interactions indicate stronger impact for the greater downstream effect. When the ligand (PAHs) can bind to the protein (AhR) with a strong interaction, it can result in a greater damage of DNA transcription, which can lead to tumorigenesis and eventually to cancer. The heterodimer of PAHs-AhR and ARNT will bind to the XRE (xenobiotic responsive element) (where the heterodimer is recognized as a xenobiotic). This 
heterodimer binding will control the modulation of the target expression which can lead to cancer [2].

\section{CONCLUSION}

$\mathrm{BaP}$ was the strongest carcinogenic PAHs compound, followed by BaA and PA due to its strong interaction with AhR. It might have a higher risk leading to cancer with adverse effects compared to the $\mathrm{BaA}$ and PA interaction to AhR.

\section{ACKNOWLEDGEMENTS}

We would like to acknowledge Brawijaya University as our university who allows us to conduct this research. We would like to thank to Prof. Fatchiyah, Ph.D., Nia Kurniawan, S.Si, M.P., D.Sc., and Yoga Dwi Jatmiko, S.Si., M.App.Sc., $\mathrm{Ph} . \mathrm{D}$. as our bioinformatics lecturers and the laboratory assistants for their guidance and feedbacks on our project.

\section{REFERENCES}

1. ATSDR, Environmental Medicine; Environmental Health Education (2011).
"Toxicity of Polycyclic Aromatic Hydrocarbons (PAHs): Health Effects Associated With PAH Exposure". Retrieved April 8, 2019, from Agency for Toxic Substances \& Disease Registry:

https://www.atsdr.cdc.gov/csem/csem.asp?cse $\mathrm{m}=13 \& \mathrm{po}=11$

2. Larigot, L., Ludmila, J., Julien, D., dan Xavier, C., Biochem Open, 2018, 7, 1-9.

3. U.S. National Library of Medicine. (2017, September 5). HSDB: Polycyclic Aromatic Hydrocarbons. Retrieved April 1, 2019, from Toxicology Data Network: http://toxnet.nlm.nih.gov/cgibin/sis/search2/r?dbs+hsdb: @term+@DOCNO $+7092$

4. Parsegian, Vozken A., Journal of Statistical Physics, 2006, 123(3), 709-710.

5. Maharramov, A.M. Mahmudov, K.T., Kopylovich, M.N., Pombeiro, A.J.L., Noncovalent Interactions in the Synthesis and Design of New Compounds, 2016, John Wiley \& Sons, Inc, New Jersey.

6. Bersten, D.C., Sullivan, A.E., Peet, D.J., Whitelaw, M.L., Nat Rev Canc, 2013, 13, 827841. 